\title{
Analysis of wind farm islanding experiment
}

Pedersen, Jørgen Kaas; Pedersen, Knud Ole Helgesen; Poulsen, Niels Kjølstad; Akke, Magnus

Published in:

IEEE Transactions on Energy Conversion

Link to article, DOI:

$10.1109 / 60.849125$

Publication date:

2000

Document Version

Publisher's PDF, also known as Version of record

Link back to DTU Orbit

Citation (APA):

Pedersen, J. K., Pedersen, K. O. H., Poulsen, N. K., \& Akke, M. (2000). Analysis of wind farm islanding experiment. IEEE Transactions on Energy Conversion, 15(01), 110-115. https://doi.org/10.1109/60.849125

\section{General rights}

Copyright and moral rights for the publications made accessible in the public portal are retained by the authors and/or other copyright owners and it is a condition of accessing publications that users recognise and abide by the legal requirements associated with these rights.

- Users may download and print one copy of any publication from the public portal for the purpose of private study or research.

- You may not further distribute the material or use it for any profit-making activity or commercial gain

- You may freely distribute the URL identifying the publication in the public portal

If you believe that this document breaches copyright please contact us providing details, and we will remove access to the work immediately and investigate your claim. 


\title{
Analysis of Wind Farm Islanding Experiment
}

\author{
Jørgen Kaas Pedersen, Magnus Akke, Member, IEEE, Niels Kjølstad Poulsen, and Knud Ole Helgesen Pedersen
}

\begin{abstract}
This paper deals with the problems related to an islanding experiment performed at Rejsby Hede in Denmark. During the experiment several interesting observations were made in connection to distortion of voltages and currents. Observations were also made in connection to variation of frequency and phase. In this paper the data are analyzed in three different manners and the results are related to the physics of the electric system.
\end{abstract}

Index Terms-Determination of distortion, frequency estimation, inductions machines, islanding, wind farm.

\section{INTRODUCTION}

A S EARLY as 1918, C. P. Steinmetz considered wind power as very interesting [1]. He foresaw the use of induction generators attached to the wind-wheel, feeding the power into a larger bulk system with hydro and steam cogeneration. Today, after $80 \mathrm{yr}$, wind power has been successfully introduced in Denmark. Further expansion plans will turn wind power into a major production source in Denmark. At remote windy locations, where the grid can be weak, wind mills are clustered together to wind farms. One special characteristic of wind power is the variability in power generation. The expansion plans call for tools to analyze voltage and frequency behavior, both at synchronized and islanding operation. Paper [2] describes the dynamics and stability of both synchronous and induction wind turbine generators. As reported in [3] there is also a risk for self-excitation at islanding operation of induction generators with capacitive compensation. Paper [4] is a survey of wind turbine integration into a weak grid.

In this paper we use measurement data from an experiment where a wind farm was forced into an islanding situation. The test is part of a larger EU-project to test an Advanced Static Var Compensator (ASVC, see [5] or [6]). The measurement noise, harmonics, phase jump in voltage at islanding make the data useful to benchmark frequency estimation algorithms.

The islanding experiment presented in this paper is interesting from two perspectives. In the narrow perspective it is important to ensure that nearby customers to this specific wind plant are not disturbed. In a broader perspective it is also important to understand the underlying dynamics behind the frequency and voltage behavior after disconnection.

A relevant question is how to interpret the term "frequency" at islanding. Do we implicitly mean that frequency always refers to rotor speed, or shall we strictly define frequency

Manuscript received June 16, 1998; revised October 22, 1998.

J. K. Pedersen and K. O. H. Pedersen are with the Department of Electric Power Engineering, Build. 325, Technical University of Denmark, DK-2800 Lyngby, Denmark.

M. Akke is with SYDKRAFT, Carl Gustavs Väg 1, S-20509 Malmö, Sweden.

N. K. Poulsen is with the Department of Mathematical Modelling, Build. 321,

Technical University of Denmark, DK-2800 Lyngby, Denmark.

Publisher Item Identifier S 0885-8969(00)02221-X

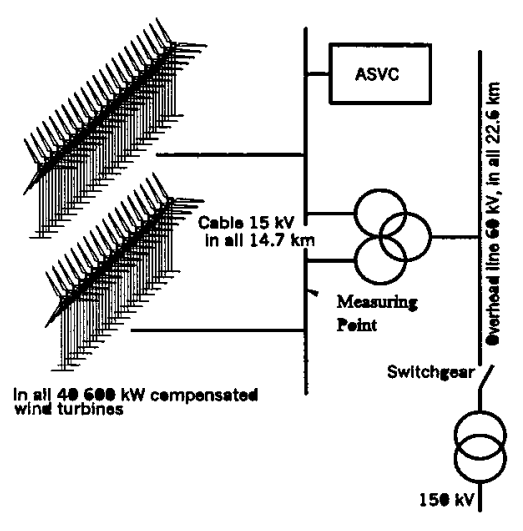

Fig. 1. Situation plan for Rejsby Hede wind farm.

as the derivative of the phase angle? At islanding the two interpretations give very different answers.

One of the paper's purposes is to show that independent methods give consistent results. This is important since the analysis is based on data measurements from the real world and the "true" frequency is unknown.

Typical use of frequency estimation in power systems is in protection schemes against loss of synchronism, under- or overfrequency relaying, input signal for power system stabilization and as a part of spectral estimation for periodic signals.

The material in the paper is organized as follows. Section II provides background information on the wind farm, the island field test and the measuring system. Section III presents a preliminary analysis of the recorded data. Section IV shows how outliers rejection can be used to get a smooth frequency estimate that corresponds to the rotor speed. Section $\mathrm{V}$ uses a parametric approach where frequency and harmonic content are estimated simultaneously. Here frequency is strictly interpretated as the rate of change in phase angle.

\section{BACKGROUND}

\section{A. Description of the Windfarm}

The islanding experiment was carried out on a wind farm in Denmark at Rejsby Hede, located in the south of Jutland. The wind farm, with 40 wind turbines, its electrical surroundings, and an ASVC is shown in Fig. 1. To understand what happens after islanding, we have to consider the balance of reactive power of the generators, the internal cabling and the external overhead line. Each generator $(600 \mathrm{~kW} / 690 \mathrm{~V} / 4$ poles induction machine) has a no-load reactive power consumption of $200 \mathrm{kVar}$, which partly is balanced by a compensating capacitor producing $150 \mathrm{kVar}$. A $690 \mathrm{~V} / 15 \mathrm{kV}$ transformer on each generator has a reactive power consumption of $1 \mathrm{kVar}$, which is negligible. 

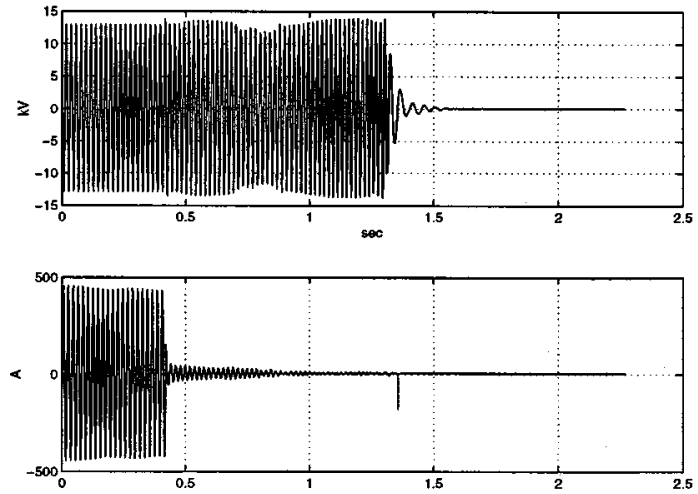

Fig. 2. Voltage and current in one phase.

Internal to the wind farm, there are three different types of $15 \mathrm{kV}$ cables used between the generators and the bus bars. In all $14.7 \mathrm{~km}$ of cables with a total capacitance of $4.32 \mathrm{uF} / \mathrm{ph}$ ase equal to $305 \mathrm{kVar}$. The $22.6 \mathrm{~km}$ overhead line from the wind farm site to the switch gear adds around $150 \mathrm{kVar}$. When the switch gear is tripped and the wind farm is islanding the total demand for reactive power at $50 \mathrm{~Hz}$ amounts 1.6 MVar. In other words, the wind farm is $80 \%$ compensated which should prevent self excitation from taking place. These considerations have not taken the ASVC into account. During normal operation of the wind farm the ASVC produces the lacking reactive power. But the ASVC was switched off at the moment of islanding and had no influence on what happened afterwards.

\section{B. The Measuring System}

Voltage and current in 3-phases were measured during the islanding experiment. The measurements started a short time before the connection to the grid was switched off and continued a few seconds after this disconnection. The phase voltages—around $15 / \sqrt{3}$ or $8.7 \mathrm{kV}$-were measured by special designed capacitive voltage dividers to assure satisfactory frequency characteristic. The measuring system consists of a PC equipped with a $300 \mathrm{kHz}, 12$-bit, multichannel A/D-converter and as front-end a signal conditioning circuit including an antialiasing filter. The sampling rate was set to $12.8 \mathrm{kHz}$, or 256 measurements during a $50 \mathrm{~Hz}$ cycle.

\section{PReliminary Analysis}

Fig. 2 gives an overall view of the measurements in one phase of voltage and current. The islanding situation starts after approximately $0.4 \mathrm{~s}$. Immediately after that the current drops to one tenth of the original level and decays slowly to zero. The voltage maintains its level-more or less-after islanding, but some variations in amplitude and frequency are introduced. The voltage disappears $1 \mathrm{~s}$ later due to a forced, mechanical braking of all the wind turbines.

Fig. 3 gives a closer view of the behavior around the moment of islanding. A phase shift is observed in the voltage when islanding starts and a clear harmonic appears in the voltage after islanding. This is the fifth harmonic which before islanding is $0.4 \%$, but after islanding increases to $6.5 \%$ - the fifth harmonic in the current is $1.1 \%$ before islanding. The higher harmonics
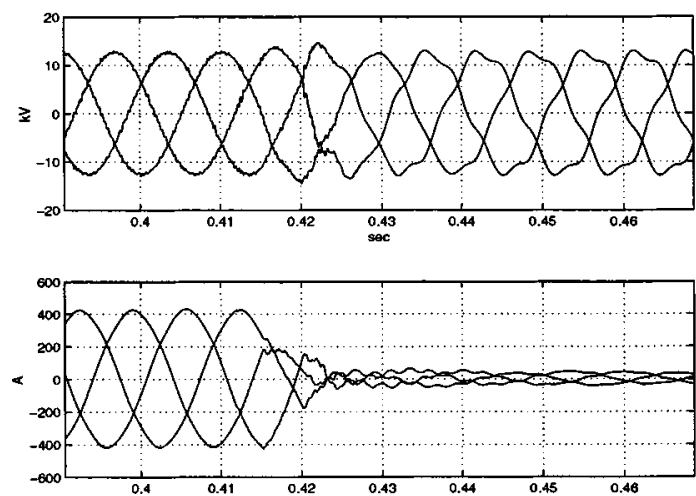

Fig. 3. Voltage and current in 3 phases around islanding.
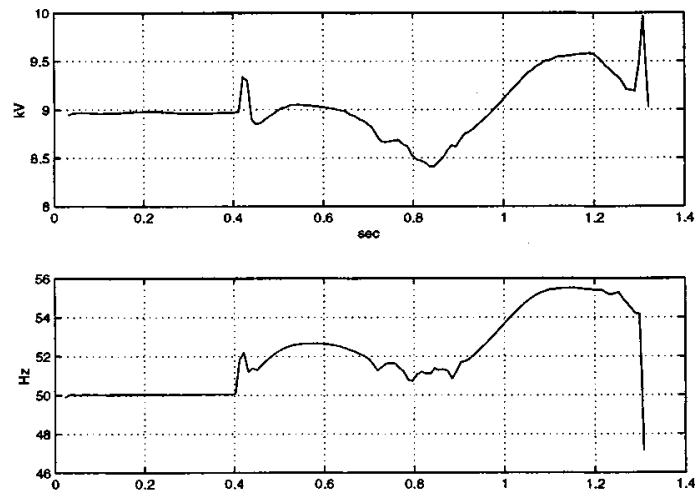

Fig. 4. RMS and frequency in one phase.

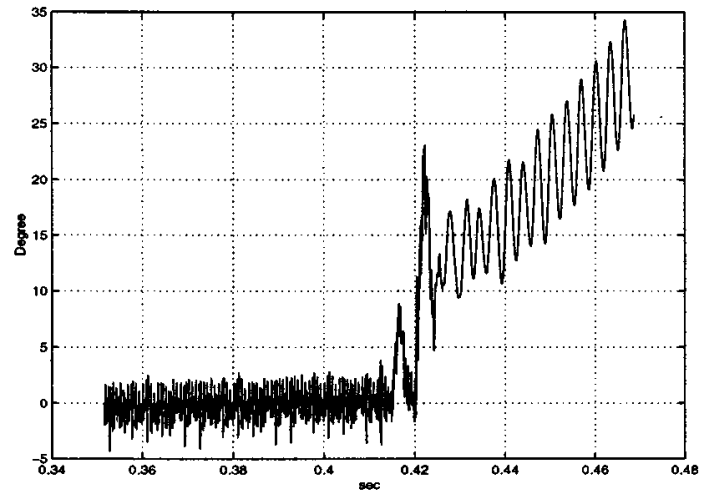

Fig. 5. Angle of space vector of voltage deviation from $50 \mathrm{~Hz}$.

seen in both voltage and current before islanding are caused by the ASVC which immediately stops operation when islanding occurs.

Fig. 4 shows the variations in RMS-value and frequency in one phase of the voltage derived from a detection of zero-crossings over the measurement period. The apparently abrupt change in frequency from $50-52 \mathrm{~Hz}$ at the time of islanding is created mainly by the phase shift. The method can not distinguish between a sudden jump in phase and frequency.

An alternative way of looking at the behavior is displayed in Fig. 5. First, the voltage space vector (a complex sinusoid or Cisoid) corresponding to the 3-phase voltages is calculated. At each sampling point the three phase quantities are converted to 
$\alpha \beta$-component by multiplying the inputs by a $2 \times 3$ matrix $A_{\alpha \beta}$, that is

$$
\left[\begin{array}{l}
v_{\alpha}(k) \\
v_{\beta}(k)
\end{array}\right]=A_{\alpha \beta}\left[\begin{array}{l}
v_{r}(k) \\
v_{s}(k) \\
v_{t}(k)
\end{array}\right]
$$

where

$$
A_{\alpha \beta}=\sqrt{\frac{2}{3}}\left[\begin{array}{ccc}
1 & -\frac{1}{2} & -\frac{1}{2} \\
0 & \frac{\sqrt{3}}{2} & -\frac{\sqrt{3}}{2}
\end{array}\right] \text {. }
$$

By interpreting $v_{\alpha}$ as real part and $v_{\beta}$ as imaginary part, we get the complex sinusoid

$$
v(k)=v_{\alpha}(k)+j v_{\beta}(k) .
$$

Next, the difference between the angle of this vector and the angle of a pure $50 \mathrm{~Hz}$ space vector is determined, and this difference is plotted in Fig. 5. It is seen that the frequency of the voltage before islanding is exactly $50 \mathrm{~Hz}$ - the "noise" is generated by higher harmonics. At the time of islanding a phase shift of approx. $10^{\circ}$ takes place-not in one go, but over most of a $50 \mathrm{~Hz}$ cycle, which may be caused by the 3 phases not being disrupted at the same time. After that the curve moves away from the $50 \mathrm{~Hz}$ case, which indicates a change in frequency. The oscillations are produced by the fifth harmonic.

\section{FREQuenCy Estimation with OUtLIER ReJeCtion}

In this section, it is assumed that power system frequency is governed by generator speed. All generators have inertia and therefore frequency is postulated to be continuous. Often voltage is preferred as input signal to frequency estimation algorithms. Voltage is advantageous since the magnitude is often close to the nominal value. In contrast, current can vary over a much broader range, and more seriously, would not work at no-load situations. Despite this the use of voltage has some drawbacks. For weak power systems, i.e., low short circuit power, sudden load changes show up as phase jumps in the voltages. Many algorithms are sensitive to these jumps and they often produce spikes in the frequency estimate.

The spikes can be characterized as statistical outliers. We propose a filter that estimates the mean and standard deviation of the frequency estimate and use this to reject the outliers. The new filter is combined with method [7] to give an algorithm more robust to phase jumps.

One way to reduce the large phase jumps is to calculate the internal voltage in the generator and use this for frequency estimation, see [8]. However, this requires current measurements and knowledge of the impedance towards the generator seen from the measurement point. Here we consider the case where only the voltage is measured and the voltage alone is used for frequency estimation. A three step procedure is described below.

First the data is prefiltered in a FIR low pass filter with crossover frequency $400 \mathrm{~Hz}$ and window length 128 . To get a sampling rate suitable for fundamental frequency estimation, the rate is decimated by a factor 8 , giving $N=32$ samples per $50 \mathrm{~Hz}$ cycle.

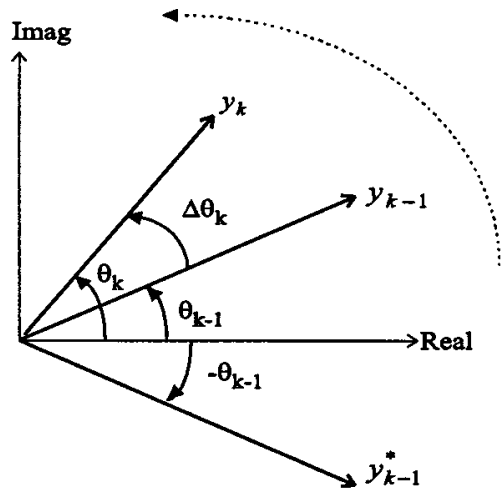

Fig. 6. Phase angles for two samples of $y$.

The idea behind the frequency estimation method [7], and many others, is to use a complex quantity and estimate the frequency from phase angle changes. The complex space vector, or complex sinusoid, is the input to the demodulation part.

\section{A. Demodulation}

The complex signal $v(k)$ from (1), is demodulated with a known complex phasor $z$ rotating at the nominal system frequency $f_{o}$ in opposition to the input. The demodulated phasor $y$ is calculated as,

$$
y(k)=v(k) z(k)
$$

with

$$
z(k)=\cos \left(-2 \pi k \frac{f_{0}}{f_{s}}\right)+j \sin \left(-2 \pi k \frac{f_{0}}{f_{s}}\right)
$$

where $f_{s}$ is the sampling frequency and $f_{o}$ is the nominal system frequency.

\section{B. Estimation of Phase Angle Change}

The phase shift of the complex variable $y$ is used to estimate the unknown frequency deviation. For each sample, $y$ rotates an angle $\Delta \theta$ as illustrated in Fig. 6.

Fig. 6 shows a direct way to calculate the phase angle change $\Delta \theta$ per sample, that is

$$
\Delta \theta=\arg \left(y_{k}\right)-\arg \left(y_{k-1}\right) .
$$

However this can cause problem with large phase jumps when the angles cross over 180 or $360^{\circ}$ borders. One simple way to avoid these problem is to calculate $\Delta \theta$ from

$$
\Delta \theta=\arg \left(y_{k} \cdot y_{k-1}^{*}\right)
$$

where $*$ is used for complex conjugate.

\section{Postfiltering with Outlier Rejection}

Fig. 7 shows the estimate after being postfiltered in a third order Butterworth low-pass filter with a cut-off frequency at $150 \mathrm{~Hz}$.

Fig. 7 shows a large frequency spike at $t=0.43 \mathrm{~s}$ caused by the phase jump when the wind farm is disconnected from the external grid. This is not a frequency variation caused by changing generator speed. When frequency is interpretated as change in rotor speed this spike should be filtered away. One way is to use a linear filter with a large time constant. However, 


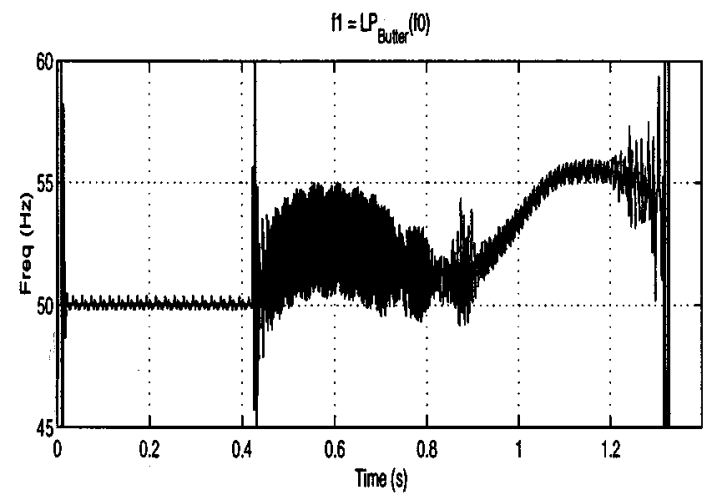

Fig. 7. Frequency estimate after third order Butterworth LP filter.

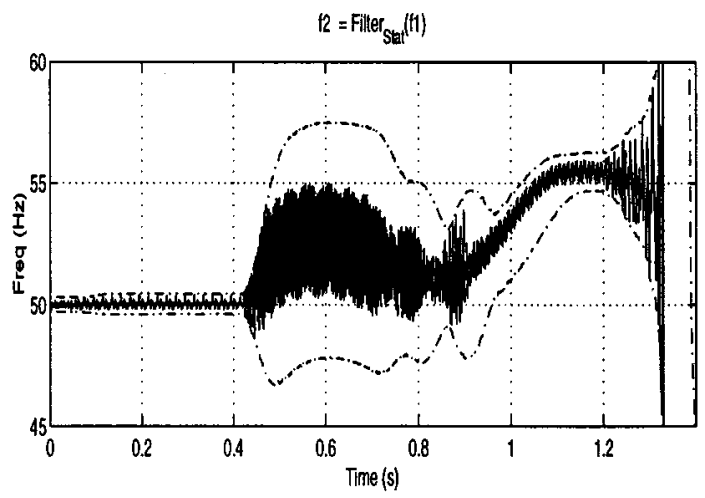

Fig. 8. Frequency estimate after outlier rejection filter (solid) and the rejection bounds (dash-dot).

this slows down the algorithm. An alternative approach has been tested where the spikes are rejected by characterizing them as statistical outliers. The standard deviation of the frequency estimate is calculated for a sliding window with length $N$. The equation used is

$$
\sigma_{f}(k)=\sqrt{\frac{1}{N-1} \sum_{i=k-N+1} \Delta f_{i} \Delta f_{i}^{*}}
$$

where $\Delta f_{i}$ is the deviation from the window mean,

$$
\Delta f_{i}=f_{i}-\bar{f}
$$

and the window mean value $\bar{f}$ is

$$
\bar{f}=\frac{1}{N} \sum_{i=k-N+1}^{k} f_{i} .
$$

One way to avoid extreme values at abnormal conditions, such as zero input, is to limit $\sigma_{f}$ to be within a reasonable range, $\sigma_{f \min }<\sigma_{f}<\sigma_{f \max }$. Outliers are rejected by putting a bound on each new frequency estimate. It has to be within the interval $\bar{f} \pm 3 \sigma_{f}$, that is

$$
f_{k} \leftarrow \max \left[\min \left[f_{k}, \bar{f}+3 \sigma_{f}\right], \bar{f}-3 \sigma_{f}\right] .
$$

The output from the Butterworth filter shown in Fig. 7 has been filtered in this way. The window length was $N=96$ and the standard deviation $\sigma_{f}$ was limited between 0.1 and $5 \mathrm{~Hz}$. The result is shown in Fig. 8 together with the bounds.

Fig. 8 shows that the outlier rejection filter eliminates the large spikes at around $0.43 \mathrm{~s}$.

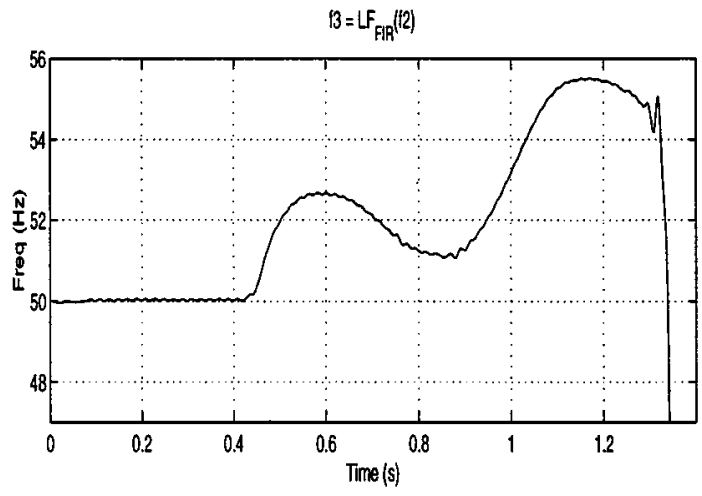

Fig. 9. The resulting frequency estimate after low-pass, outlier rejection and final low-pass filtering.

As a final step, the estimate goes into a FIR low-pass filter designed in Matlab with $f_{c}=20 \mathrm{~Hz}$ and $N=38$. Fig. 9 shows the result in a frequency estimate.

\section{PARAmetric Estimation}

Three different types of methods have been applied in order to consolidate the interpretations. The last one presented here is based on a simple parametric statistical method. The basic assumption in this contexts is that the signals involved are periodic and it is possible to Fourier expand the signals. More precisely it is assumed that a model

$$
y_{k}^{m}=\hat{y}_{k}^{m}+e_{k}^{m} \quad \text { where } \quad m=r, s, t
$$

where

$$
\hat{y}_{k}^{m}=\sum_{i=0}^{n} a_{i}^{m} \cos \left(n_{i} \omega k\right)+b_{i}^{m} \sin \left(n_{i} \omega k\right)
$$

is valid for each phase ( $y_{k}^{m}$ is the voltage or current in one phase). Here $k$ is the time index and $e_{k}^{m}$ is a zero mean noise. The coefficients $a_{i}^{m}, b_{i}^{m}$ contain information on the amplitude $A_{i}^{m}$ and the phase (with respect to origin) $\phi_{i}^{m}$ of each harmonic component in each phase, i.e., $\hat{y}_{k}^{m}=\sum_{i=0}^{n} A_{i}^{m} \sin \left(n_{i} \omega k+\phi_{i}^{m}\right)$. The basis assumption concerning the periodicallity of the signals are, according to Fig. 3, clearly not fulfilled in the islanding point, which is not the same for all three phases. The model (2) might also be written as

$$
\left[\begin{array}{lll}
y_{k}^{r} & y_{k}^{s} & y_{k}^{t}
\end{array}\right]=x_{k}^{\top} \theta+\left[\begin{array}{lll}
e_{k}^{r} & e_{k}^{s} & e_{k}^{t}
\end{array}\right]
$$

where

$$
x_{k}=\left[\begin{array}{c}
\cos (\omega k) \\
\sin (\omega k) \\
\cos (2 \omega k) \\
\vdots \\
\sin \left(n_{n} \omega k\right)
\end{array}\right] \quad \theta=\left[\begin{array}{ccc}
a_{1}^{r} & a_{1}^{s} & a_{1}^{t} \\
b_{1}^{r} & b_{1}^{s} & b_{1}^{t} \\
a_{2}^{r} & a_{2}^{s} & a_{2}^{t} \\
\vdots & & \\
b_{n}^{r} & b_{n}^{s} & b_{n}^{t}
\end{array}\right] .
$$

If the frequency $\omega$ is known then it is a standard task to make a estimate of the coefficients in (2) which minimize the sum of squares,

$$
J-\frac{1}{2} \sum_{j=1}^{N} \sum_{m}\left(y_{k_{j}}^{m}-\hat{y}_{k_{j}}^{m}\right)^{2}
$$



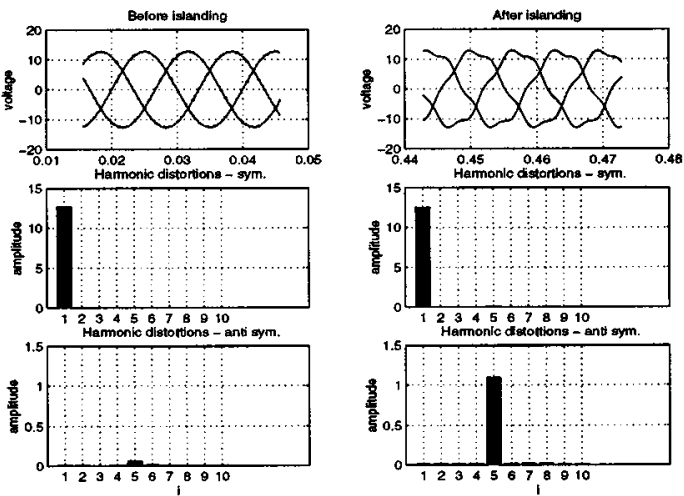

Fig. 10. The contents of higher harmonic components in the voltage before and after islanding.

over a data window of length $N$. Such an LS-estimate is simply given by

$$
\hat{\theta}=\left(X^{\top} X\right)^{-1} X^{\top} Y
$$

where

$$
X=\left[\begin{array}{c}
x_{1}^{\top} \\
\vdots \\
x_{N}^{\top}
\end{array}\right] \quad Y=\left[\begin{array}{ccc}
y_{1}^{r} & y_{1}^{s} & y_{1}^{t} \\
& \vdots & \\
y_{N}^{r} & y_{N}^{s} & y_{N}^{t}
\end{array}\right] .
$$

On the other hand, if the frequency $\omega$ is unknown, it has to be estimated too. If we use the LS criterion (4) and define

$$
\begin{aligned}
g_{l} & =\frac{\partial J}{\partial \omega}=-\sum_{i=1}^{N} \sum_{m} \frac{\partial \hat{y}_{k}^{m}}{\partial \omega}\left(y_{k}^{m}-\hat{y}_{k}^{m}\right) \\
H_{l} & =\frac{\partial^{2} J}{\partial \omega^{2}}=\sum_{i=1}^{N} \sum_{m}\left(\frac{\partial \hat{y}_{t}^{m}}{\partial \omega}\right)^{2}-\frac{\partial^{2} \hat{y}_{k}^{m}}{\partial \omega^{2}}\left(y_{k}^{m}-\hat{y}_{k}^{m}\right) .
\end{aligned}
$$

[The derivatives can easily be found using (2)] then the estimate of $\omega$ and the parameters in $\theta$ can found by utilizing a (variant of a) Newton-Raphson method, where each iteration obey

$$
\omega_{l+1}=\omega_{l}-\frac{g_{l}}{H_{l}}
$$

and (5). The approximation involved when using (5) and (6) is to neglect the cross term in the second derivative. There exists a recursive version of this method, which turns out to be a kalman filter mechanization, and is well suited for real time processing. In a post mortem analysis (as used in this paper) the algorithm is directly applicable with some rectangular windowing techniques.

According to Fig. 3 the voltage have a high distortion component after islanding. In order to investigate this the parameters in (2) are estimated up to the order of 10 . The results are shown in Fig. 10, where it is clear that the fifth harmonic is rather significant after islanding. This is also clear from Fig. 11 where the variations of the amplitude of the first (i.e., base frequency) and fifth harmonic in the voltage are shown. The estimate of the parameters in (2) are based on data from a time window with a length corresponding to 1.25 of a cycle (at $50 \mathrm{~Hz}$ ).

Similarly, before islanding there exist significant components in the currents, as shown in Fig. 12.

At the point of islanding the frequency and phases change instantaneously due to the lack of electric momentum. Since
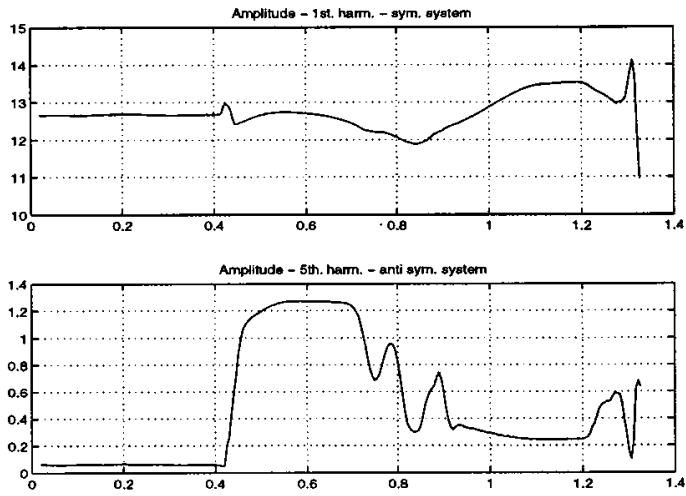

Fig. 11. The amplitude of the first and fifth harmonic component.
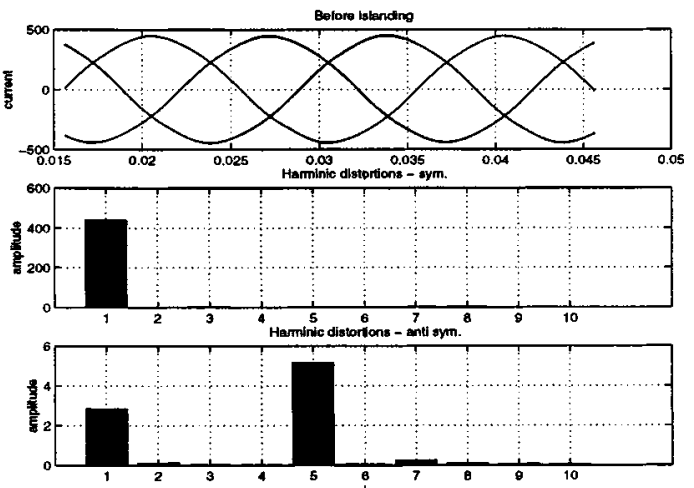

Fig. 12. The contents of higher harmonic components in the current before islanding.

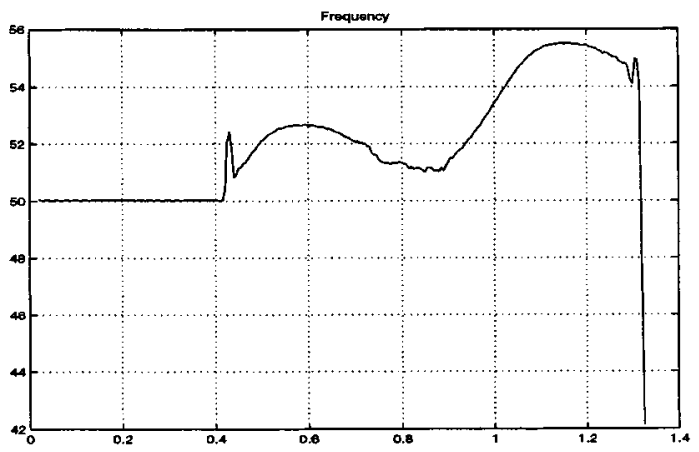

Fig. 13. The estimated frequency as function of time.

the wind turbine system accelerates the frequency and phases vary. This is shown in Fig. 13, where some oscillation in the mechanical system is obvious.

In order to investigate the instantaneous changes in frequency and phase the analysis is performed just before and just after the islanding. The result of the analysis is an estimate of the basic frequency $\omega_{b}$ and $\omega_{a}$ (before and after) and phase $\phi_{b}$ and $\phi_{a}$ (with respect to origin) of the first harmonic in the symmetric system. The analysis shows a jump in frequency of $1.15 \mathrm{~Hz}$. The phase jump can be estimated by:

$$
\phi=\left(\omega_{a} t_{i s}+\phi_{a}\right)-\left(\omega_{b} t_{i s}+\phi_{b}\right)
$$

where $t_{i s}$ is the instant of islanding. The phase jump in the reported experiment is $7.5^{\circ}$. 


\section{DISCUSSION}

The above analyses focus on the behavior of the voltage during and after the moment of islanding. The reason for the behavior can be traced back to the electrical and mechanical properties of the system. A phase shift in the voltage must be expected when islanding occurs. The size of the shift can be calculated when knowing the characteristic data of the generators and the impedances in the grid and knowing that the generators were $75 \%$ loaded just before islanding. A phase shift of approximately $12^{\circ}$ is found which is in good agreement with the measurements. Since the generators go from an almost full-load to a no-load condition at the moment of islanding, then a shift in frequency takes place. However, this is just a minor shift due to the low slip of the generators. After islanding, the wind turbines start accelerating the generators which explains the increase in frequency observed in the measurements. The increase in frequency is superimposed with a slow oscillation which might be caused by torsion in the shaft connecting two inertia loads-on one side the wings of the turbine, and on the other side the rotor of the generator. It is surprising that the voltage does not fall away after islanding, since the generators are under-compensated. Immediately after islanding the voltage decreases, but after a short while it starts increasing again-following the variations in the frequency. We have no sure explanation to this phenomena, but it might be caused by the capacitance of the internal cabling and of the $22 \mathrm{~km}$ long overhead line from the wind farm site to the line breaker. An over-compensation takes place if just 8 wind turbines-or less-are running due to this extra capacitance. Such a situation can arise if the brakes on all wind turbines are not activated at the same time. According to specifications breaking is initiated $0.2 \mathrm{~s}$ after a frequency higher than $51.5 \mathrm{~Hz}$ is detected. This seems not to be the case and this point has to be examined closer in future experiments. The fifth harmonic appearing clearly in the voltage after islanding is produced by the generators. Before islanding this fifth harmonic component is suppressed because the wind farm is connected to a "strong" low impedance power system.

\section{CONCLUSIONS}

When planning a wind farm, precautions should be taken against islanding - to prevent disastrous consequences for components connected to the islanding network. The experiment has demonstrated that the behavior around islanding is quite different from what normally is seen in a power system. Islanding starts with a sudden phase jump followed by changes in voltage level and frequency and significant harmonics are appearing. New tools are necessary to analyze islanding. Different mathematical approaches have been tested in this paper and they all lead to consistent results. Thus, identifying islanding from measurements of the 3-phase voltage is possible and suitable algorithms can be derived. The results have attracted attention among Danish power utilities and wind turbines manufactors and we have been encouraged to continue the study. A mobile measuring system have been designed. New experiments and further experiences will be gained in the future.

\section{REFERENCES}

[1] C. P. Steinmetz, "America's energy supply," in Proc. of the AIEE., vol. XXV, 1918, pp. 985-1014.

[2] E. N. Hinrichsen and P. J. Nolan, "Dynamics and stability of wind turbine generators," IEEE Trans. on Power Apparaturs and Systems, vol. PAS-101, no. 8, pp. 2640-2648, Aug. 1982.

[3] G. L. Park and O. W. Zastrow, "Interconnection issues concerning consumer-owned wind electric generators," IEEE Trans. on Power Apparaturs and Systems, vol. PAS-101, no. 7, pp. 2375-2382, July 1982.

[4] T. S. Davies, R. Mayer, and H. Nouri, "Integration of wind turbines into the low voltage networks," in 30th Universities Power Engineering Conference, vol. 2, 1995, pp. 567-570.

[5] J. Kaas Pedersen, "Compensating of reactive power from wind turbines with power electronics equipment," in EWEA Special Topic Conference on Integration of Wind Power Plants in the Environment and Electrical Systems, Rom, 1996.

[6] K. Bergmann, K. Renz, F. Schettler, R. Stöber, H. Tyll, M. Weinhold, K. Søbrink, N. Jenkins, and J. K. Pedersen, "Application of GTO-based SVCS for improved use of the Rejsby Hede windfarm," in Ninth National Power System Conference, Kandur, 1996.

[7] M. Akke, "Frequency estimation by demodulation of two complex signals," IEEE Trans. on Power Delivery, vol. 12, no. 1, pp. 157-163, Jan. 1997.

[8] 0. Samuelsson, "Power system damping — Structural aspects of controlling active power," Ph.D. dissertation, Department of Industrial Electrical Engineering and Automation, Lund Institute of Technology, Lund, Sweden, 1997.

[9] C. P. Steinmetz, “America's energy supply," Proc. of the IEEE, vol. 86, no. 4, pp. 723-734, Apr. 1998.

Jørgen Kaas Pedersen was born in a village near Copenhagen, Denmark, on March 22, 1935. He graduated with a Masters degree in applied physics in 1961. After working eight years in Danish industries, he was employed at the Technical University in a department for electric technique. His interest for power electronics increased, especially for use of power electronics in the grid. He is now employed in the Department of Electric Power Engineering and he is working with ASVC for compensating reactive power in the grid and problems in connection with large wind farms connected to the Danish grid.

Magnus Akke (M'92) was born in Lund, Sweden, in 1961. He received the M.E.E., the Licentiate, and the Ph.D. degrees from Lund Institute of Technology, Sweden, in 1986, 1989 and 1997, respectively. He also has a Bachelors degree in business administration from Lund University, Sweden. In 1990, he started at Sydkraft, a Swedish power utility where his work tasks are power system analysis and relay protection. He has been a visiting Scientist at University of Newcastle, Australia, and at Cornell University, USA.

Niels Kjølstad Poulsen was born in the central part of Sjaelland, Denmark in 1956. He received his M.Sc. and Ph.D. degrees in electrical engineering from the Institute of Mathematical Statistics and Operations Research (IMSOR), at the Technical University of Denmark, in 1981 and 1984, respectively. He has been employed at the Technical University of Denmark since 1984, and since 1990 he has been an Associate Professor at the Department of Mathematical Modelling. His primary research interests are within stochastic control theory, system identification, recursive estimation, and adaptive control.

Knud Ole Helgesen Pedersen was born in Copenhagen, Denmark in 1943. He received the M.Sc. degree in electrical engineering in 1967 and the Ph.D. degree in automatic control in 1970, both from the Technical University of Denmark. From 1970 to 1973 he worked at CERN, Geneva, and from 1973 to 1985, he worked in Danish industry as a Research Engineer. During that time he was mainly occupied with digital process control. Since then, he has been an Associate Professor at the Technical University of Denmark with his main interest in microcomputer applications in the power system. 